\title{
Speciation and Antimycotic Susceptibility Pattern of Candida Species Isolated from Various Clinical Specimen by Using Chromogenic Agar and Conventional Method
}

\author{
Seema Surana* and Kusumlata Lodha \\ Department of microbiology, Dr S.N.Medical College, Jodhpur Rajasthan India \\ *Corresponding author
}

\section{A B S T R A C T}

Keywords

Antimycotic susceptibility,

Candida,

Chromogenic

agar

Article Info

Accepted:

07 November 2018

Available Online:

10 December 2018
A total of 100 isolates of candida species were recovered from various clinical samples. C. albicans was the most common isolated species (42\%) followed by $C$. krusei (17\%) C. tropicalis (15\%) C. parapsilosis (13\%) C. famata (8\%) C. glabrata (4\%) and Cryptococcus species (1\%). Non albicans Candida were isolated at a higher rate $(58 \%)$ than $C$. albicans. Most of the Candida isolate were susceptible to Amphotericin (81\%) followed by Nystastin (79\%) and Miconazole (58\%). Fluconazole was least effective with $79 \%$ resistant. So goal of the study was to show there is an increase in the incidence of No nalbicans Candida with antifungal resistant strain of Candida species underlines the need of early and accurate diagnosis of infecting Candida species along with antifungal susceptibility testing for selecting the most appropriate antifungal agent for therapy.

\section{Introduction}

Over the last few years, the incidence of mycotic infection has progressively increased. Fungi once considered as non-pathogenic or less virulent are now recognized as primary cause of morbidity and mortality in immunecompromised and severely ill patients Mokaddas et al., (2007).

Candidiasis is the commonest fungal disease found in human. The infection may be acute or chronic, superficial or deep and its clinical spectrum is wide. It is found mainly as secondary infection in individual with some underlying immune compromised condition and very rarely as primary disease.

Non albicans Candida species are emerging pathogens and can also colonize human mucocutaneous surfaces and invades tissues, leading to life threatening disease in patients whose cell mediated immunity is decreased by disease or iatrogenic intervention (Ajello, 1997; Verma, 2003; Akpam, 2002 and AlAbeid et al., 2004, prolonged use of antimicrobial drugs, diabetes, chemotherapy and catheterization (Ali Zarei, 2013; Anil K Paswan, 2012; Anil, 1997 and Baradkar et al., 1996). 
Although $C$. albicans remains the most common cause of human candidiasis, now for the past four decades Non albicans Candida species like $C$. glabrata, $C$. krusei, $C$. parapsilosis, $C$. tropicalis, $C$. rugosa are emerging as important opportunistic pathogens which have shown increased resistance to anti-fungal agents Abi-said et al., (1997). These Candida species differ in their expression of putative virulence factors and antifungal susceptibility Baillie and Douglas (1998).

Accurate species identification is therefore important for the treatment of Candida infection as the Non albicans Candida species of Candida continued to be increasingly documented as not all the species respond to the same treatment.

This study has been undertaken to isolate and Speciated Candida species from the various clinical specimens and to study the distribution of Candida albicans and Non albicans Candida species in clinical specimens and to determine the anti-fungal susceptibility pattern of Candida species.

\section{Materials and Methods}

The study was conducted in the Microbiology lab of Mahatma Gandhi Hospital Dr. S.N. Medical College, Jodhpur. A total of 100 Candida species isolated from various clinical specimens including urine, pus, sputum, stool and bronchoalveolar lavage (BAL) were taken up for the study over a period of one year from out patients and in patients admitted into various wards and intensive care units.

\section{Specimen processing}

The various clinical specimens were collected and processed as per standard microbiological guidelines. The primary inoculation of specimens was done on Blood agar and MacConkey agar medium. The culture plates were incubated aerobically at $37^{0} \mathrm{C}$ for $24-48$ hours. The visual growth is stained and one which revealed gram positive budding yeast cells with or without pseudo hyphae were confirmed as yeast. All the isolated candida were inoculated immediately on Sabouraud Dextrose Agar (SDA) and incubated at $37^{\circ} \mathrm{C}$ for 24-48 hours. Cultures were identified by the colony characters and by gram's stain. Once the colonies were confirmed speciation done by the following methods (Segal et al., 2007; Rippon, 1988; Milne, 2007).

Germ Tube Test: (Reynolds Braude Phenomenon) (Milne, 2007; Forbes et al., 2007).

CHROM (HICHROME) Agar Candidaisolated species were inoculated on $\mathrm{Hi}$ CHROM Agar plates. These agar plates were incubated at $37^{\circ} \mathrm{C}$ for $24-48$ hours. The species were identified by characteristic colony colour as per Hi Media technical data (Sagar et al., 2013; Shyamala K. Shetter et al., 2012).

Corn meal agar inoculation-Formation of chlamydospores was identified by Dalmau plate culture method in Corn meal agar with $1 \%$ tween-80 incubated for 2- 3 days at room temperature (Ann P. Koehler et al., 1999) Observed for the presence of true hyphae or pseudohyphae, blastoconidia, arthroconidia and Chlamydospores

Sugar fermentation test (Arunaloke Chakrabarti. et al., 2008)

Sugar Assimilation Test (Arunaloke Chakrabarti et al., 2008; Larone, 2002)

Urease test.

Antimycotic Susceptibility test was done by Kirby- Bauer disc diffusion method as recommended by CLSI M-44A guidelines on methylene blue Mueller Hinton agar using commercially available.

The antifungal discs (Hi Media Mumbai, India) used for disc diffusion method were Fluconazole (10mcq), Voriconazole (1mcq), Itraconazole (10mcq), Amphotericin B 
(100mcq), Ketoconazole (10mcq), Nystatin (100mcq) and Miconazloe (50mcq). The quality control test was performed by using $C$. albican (ATCC90028) and C. parapsilosis (ATCC22019).

\section{Results and Discussion}

A total of 100 Candida species isolated from various clinical specimens like urine, sputum, pus, bronchoalveolar lavage (BAL) and stool were processed during the study period (Table $1)$.

The study showed that female $(51 \%)$ were more prone to candida infection than male (49\%) (Table 2). Consisted with study of Rizwi et al., (2011) reported female preponderance in their study group with ratio of $0.85: 1$ (M:F). Female indicating that women are at increased risk to develop UTI than men (Koneman et al., 2006). However in the study by Shanoo et al., (2017) the incidence was found to be higher in male (51\%).

In all the 100 specimens included in this study the most common clinical specimen was urine $49(49 \%)$ followed by sputum 39(39\%), pus 9(9\%), bronchoalveolar lavage (BAL) 2 (2\%) and stool 1 (1\%) (Table 3). Our observation is similar with the study of Deorukhkar et al., (2014) where urine samples were in majority (34.6\%) and Patel et al., (2012) where urine showed the highest number of isolates (34.5\%) followed by sputum $(28.9 \%)$.

In the present study Non albicans Candida were isolated at a higher rate (58\%) than Candida albicans $(42 \%)$ similar finding were observed in study by Shanoo et al., (2017) showed Non albicans Candida (58\%) and Candida albicans (42\%), Mokaddas et al., (2007) which also showed the Non albicans candida incidence $(60.5 \%)$ to be higher than that of Candida albicans (39.5\%).

Among the 58(58\%) Non albicans Candida species C. krusei 17 (17\%) C. tropicalis 15 (15\%) C. parapsilosis 13 (13\%) C. famata 8 (8\%) C. glabrata 4 (4\%) and Cryptococcus species $1(1 \%)$ were isolated (Table 4$)$. In our study $C$. krusei 17 (17\%) was most common Non albicans Candida species followed by $C$. tropicalis 15 (15\%). The present study is in agreement with study conducted by Nirmladevi et al., (2018) showed C. krusei 7 (11\%) predominant Non albicans Candida followed by $C$. tropicalis $4(6 \%)$. Whereas (Latiff et al., 2004) reported that $C$. parapsilosis was the most common Non albicans Candida species accounting for $21 \%$, Shivprakash et al., (2007) (36\%) and Enwuru et al., (2008) (18\%) documented C. tropicalis was the most common Non albicans Candida species.

Sensitivityrate for Amphotericin B, Nyststin, Miconazole, Voriconazole, Itraconazole, Ketoconazole and Fluconazole were $81 \%$, $79 \%, 75 \%, 62 \%, 48 \%, 28 \%$ and $21 \%$ respectively (Table 5). We observed that Candida albicans was less resistant to antifungal drugs compared to Non albicans Candida. Most of the Candida isolates were susceptible to Amphotericin B (81\%) and Nystatin (79\%) which is in concordance with Vijaya et al., (2011), Mondal et al., (2013). Fluconazole was least effective with only (21\%) susceptible to it which is in concordance with Ragini et al., (2011), Vijaya et al., (2011), Mondal et al., (2011).

All 100 Candida species were presumptively identified to species level by their colony morphology \& colour using chromogenic medium Mokaddas et al., (2007). 42/100 Candida species produced green coloured, 17/100 Candida species produced purple fuzzy coloured, 15/100 Candida species produced blue coloured, 13/100 Candida species produced white coloured, 4/100 Candida species produced off white coloured colonies. 
Table.1 Gender wise distributions of Candida species from various clinical specimens

\begin{tabular}{|c|c|c|}
\hline Gender & No.patients & Percentage \\
\hline Male & 49 & $49 \%$ \\
\hline Female & 51 & $51 \%$ \\
\hline
\end{tabular}

Table.2 Distribution of Candida species in various clinical specimens

\begin{tabular}{|c|c|c|}
\hline S.NO. & Clinical specimens & Total No. of isolates \\
\hline $\mathbf{1}$ & Urine & $49(49 \%)$ \\
\hline $\mathbf{2}$ & Sputum & $39(39 \%)$ \\
\hline $\mathbf{3}$ & Pus & $9(9 \%)$ \\
\hline $\mathbf{4}$ & BAL & $2(2 \%)$ \\
\hline $\mathbf{5}$ & Stool & $1(1 \%)$ \\
\hline
\end{tabular}

Table.3 Distribution of Candida species in various clinical specimens

\begin{tabular}{|c|c|c|c|c|c|c|}
\hline Species & Urine & Sputum & Pus & BAL & Stool & Total \\
& $(\mathbf{4 9 )}$ & $(\mathbf{3 9})$ & $\mathbf{( 9 )}$ & $(\mathbf{2})$ & $(\mathbf{1})$ & \\
\hline C.albicans & 17 & 22 & 3 & 0 & 0 & 42 \\
& $(34.69 \%)$ & $(56.41 \%)$ & $(33.33 \%)$ & $(0 \%)$ & $(0 \%)$ & $(42 \%)$ \\
\hline C.krusei & 9 & 7 & 1 & 0 & 0 & 17 \\
& $(18.36 \%)$ & $(17.94 \%)$ & $(11.11 \%)$ & $(0 \%)$ & $(0 \%)$ & $(17 \%)$ \\
\hline C.tropicalis & 6 & 7 & 1 & 1 & 0 & 15 \\
& $(12.24 \%)$ & $(17.94 \%)$ & $(11.11 \%)$ & $(50 \%)$ & $(0 \%)$ & $(15 \%)$ \\
\hline C.parapsilosis & 7 & 2 & 4 & 0 & 0 & 13 \\
& $(14.28 \%)$ & $(5.12 \%)$ & $(44.44 \%)$ & $(0 \%)$ & $(0 \%)$ & $(13 \%)$ \\
\hline C.famata & 6 & 0 & 0 & 1 & 1 & 8 \\
& $(12.24 \%)$ & $(0 \%)$ & $(0 \%)$ & $(50 \%)$ & $(100 \%)$ & $(8 \%)$ \\
\hline C.glabrata & 4 & 0 & 0 & 0 & 0 & 4 \\
& $(8.16 \%)$ & $(0 \%)$ & $(0 \%)$ & $(0 \%)$ & $(0 \%)$ & $(4 \%)$ \\
\hline Cryptococcus species & 0 & 1 & 0 & 0 & 0 & 1 \\
& $(0 \%)$ & $(2.56 \%)$ & $(0 \%)$ & $(0 \%)$ & $(0 \%)$ & $(1 \%)$ \\
\hline
\end{tabular}

Table.4 Species distribution of the Candida isolated from various specimens

\begin{tabular}{|c|c|c|}
\hline S.No. & Species & No. of isolates \\
\hline 1 & C. albicans & $42(42 \%)$ \\
\hline 2 & C. krusei & $17(17 \%)$ \\
\hline 3 & C. tropicalis & $15(15 \%)$ \\
\hline 4 & C. parapsilosis & $13(13 \%)$ \\
\hline 5 & C. famata & $8 \quad(8 \%)$ \\
\hline 6 & C. glabrata & $4 \quad(4 \%)$ \\
\hline 7 & Cryptococcus species & $1 \quad(1 \%)$ \\
\hline
\end{tabular}


Gram positive budding yeast cells

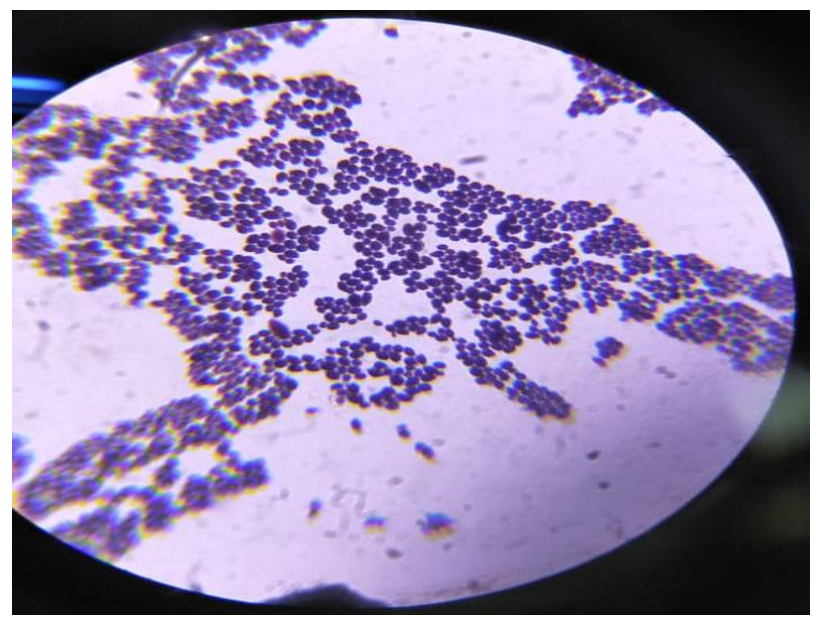

Germ Tube Test for Candida albicans

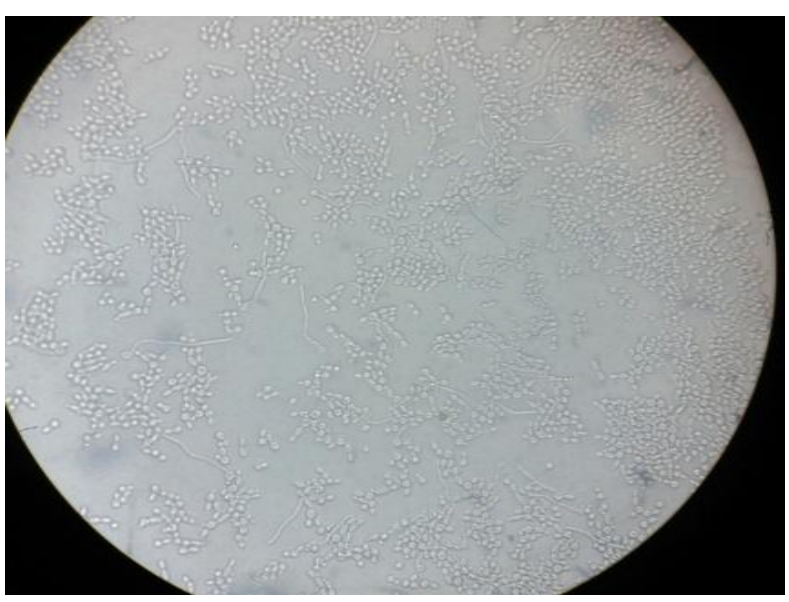

Antimycotic Susceptibility test

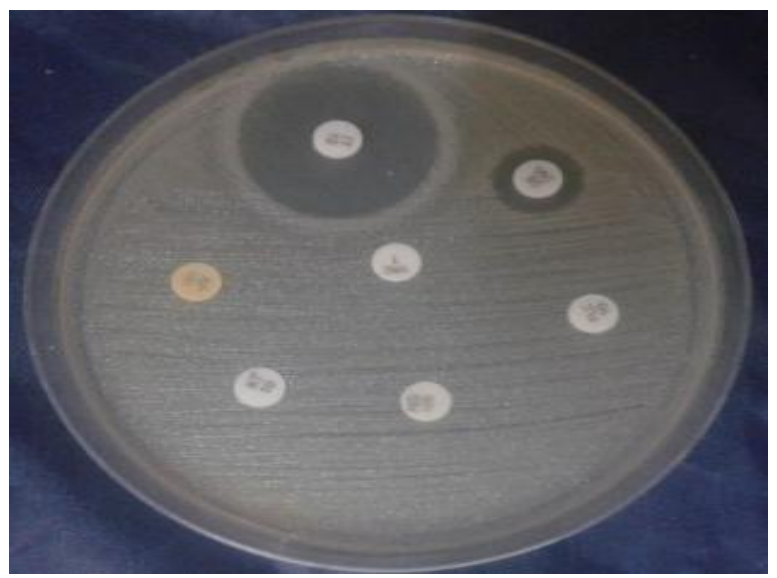



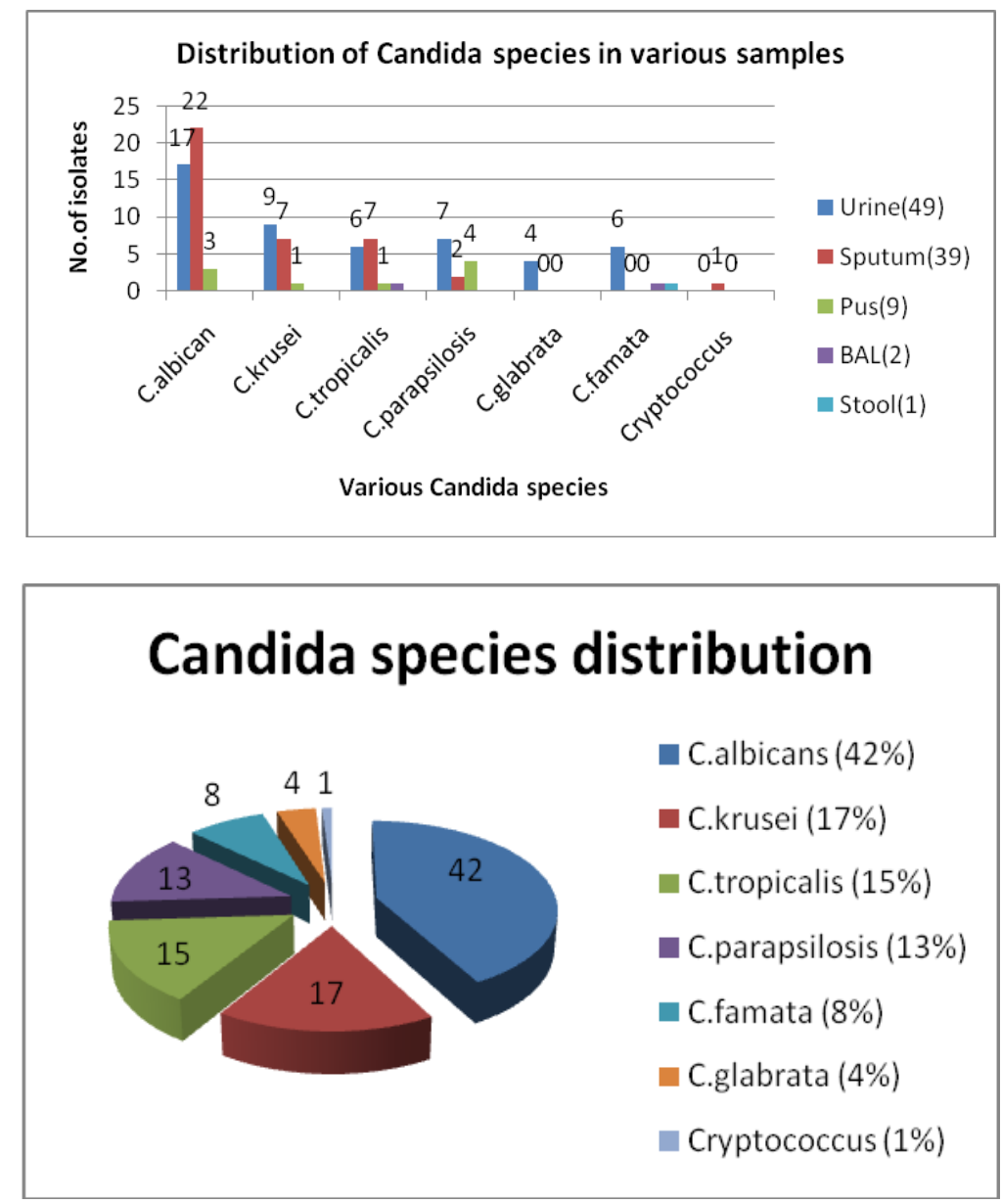

Fig.1 Morphology of Candida albicans

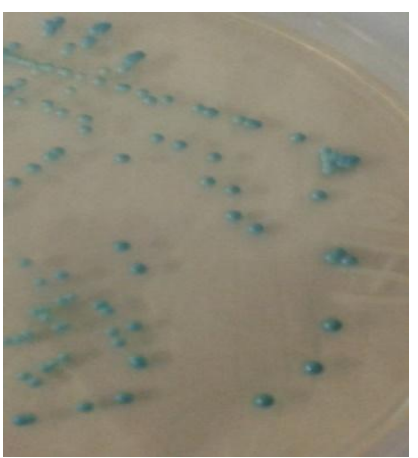

(A)

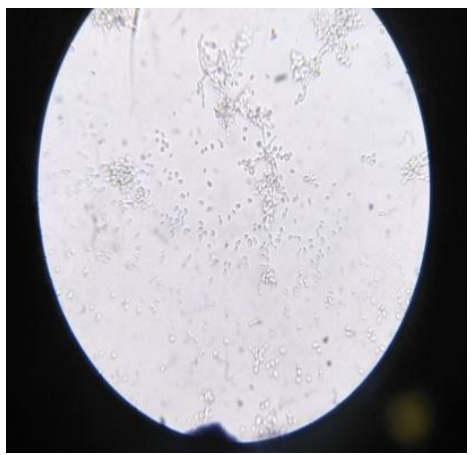

(B)

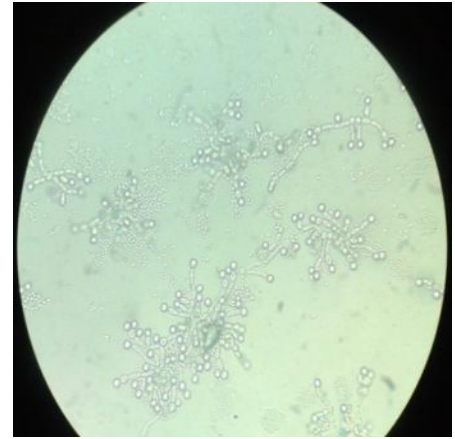

(C)

(A) Candida albicans on Hichrom agar- green, smooth colonies

(B) Candida albicans (Microscopic picture after 24 hours); Budding yeast and pseudohyphae

(C) Candida albicans (Microscopic picture after 48 hours)

Showing Chlamydospores usually terminal 
Fig.2 Morphology of C. tropicalis

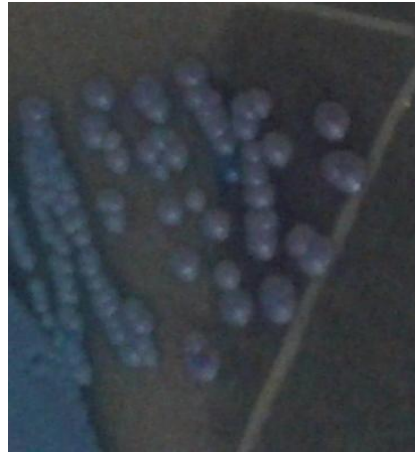

(A)

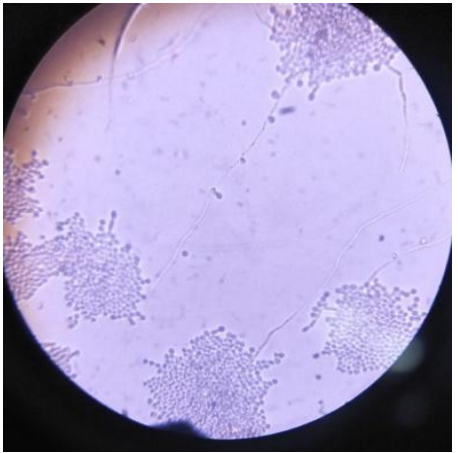

(B)

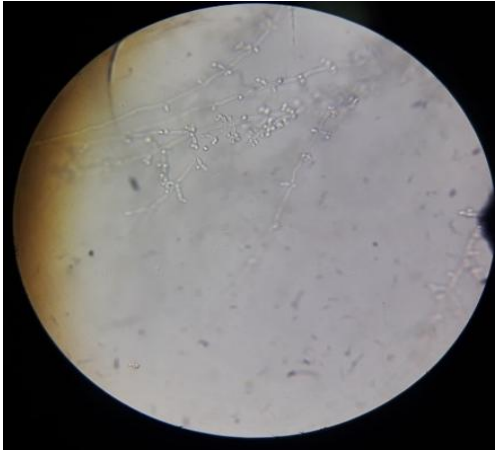

(C)

(A)Candida tropicalis on Hichrom agar: mettalic blue colonies.

(B)Candida tropicalis showing lateral blastospores (Microscopic picture after 24 hours)

(C) Candida tropicalisshowing lateral blastospores(Microscopic picture after 48 hours)

Fig.3 Morphology of C.glabrata

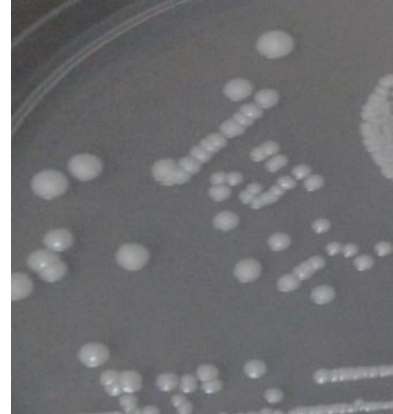

(A)

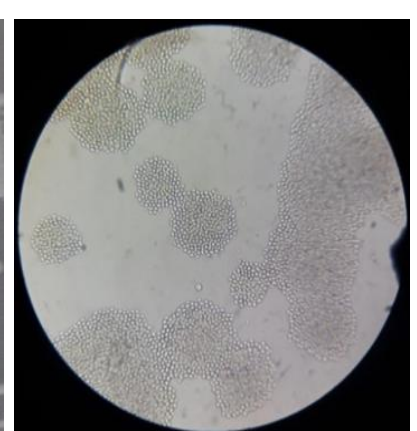

(B)

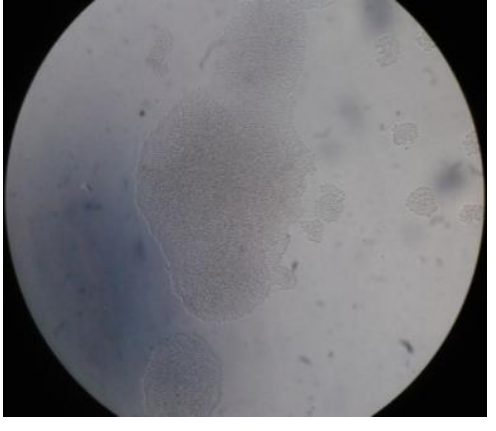

(C)

(A)Candida glabrata on Hichrom agar white small glossy colonies

(B)Candidaglabrata only small budding yeast cell (Microscopic picture after 24 hours)

(C)Candida glabrata only yeast cells no pseudo/true hyphae (Microscopic picture after 48 hours)

Fig.4 Morphology of C.parapsilosis
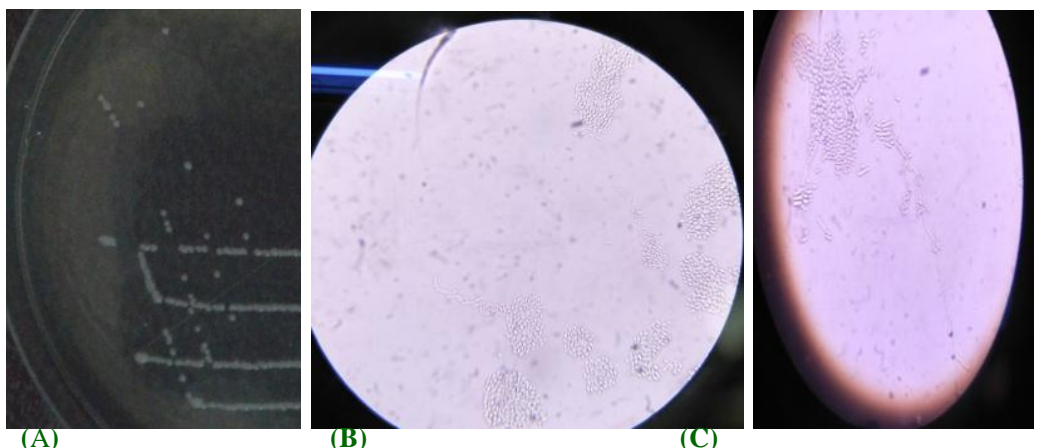

(A)Candida parapsilosis on Hichrom agar off-white large colonies;

(B) Candidaparapsilosisshort, tree like pseudohyphae.

(Microscopic picture after 24 hours)

(C) Candidaparapsilosis tree like pseudohyphae.

(Microscopic picture after 48 hours) 
Fig.5 Morphology of C.krusei

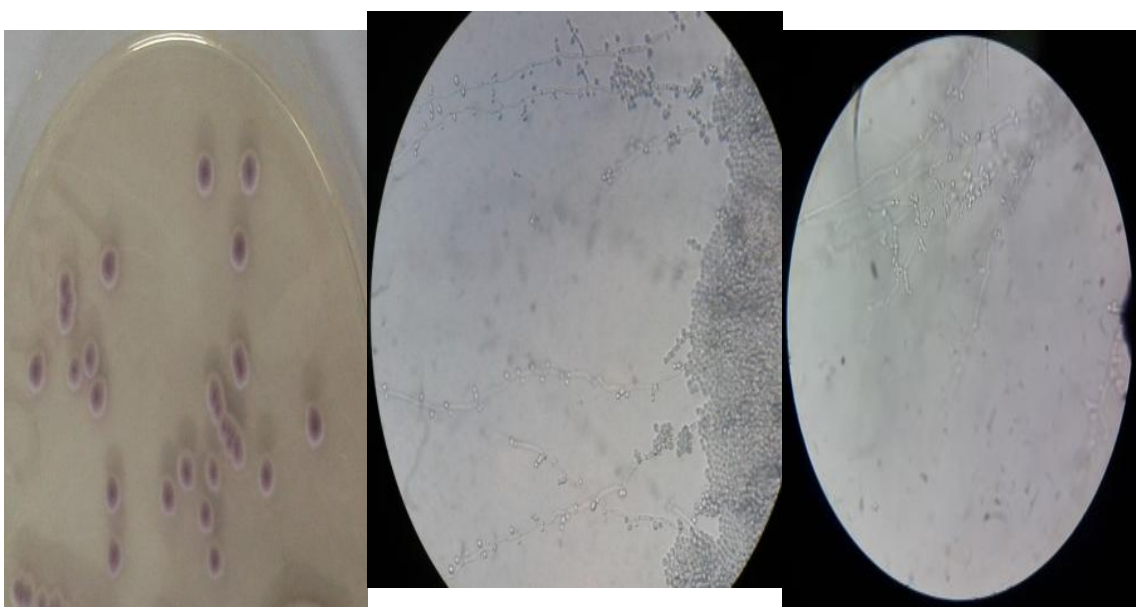

(A) (B)

(C)

(A)Candida krusei on Hichrom agar large, purple fuzzy colonies

(B)Candida kruseimatch stick appearance (Microscopic picture after 24 hours)

(C) Candida kruseimatch stick appearance (Microscopic picture after 48 hours)

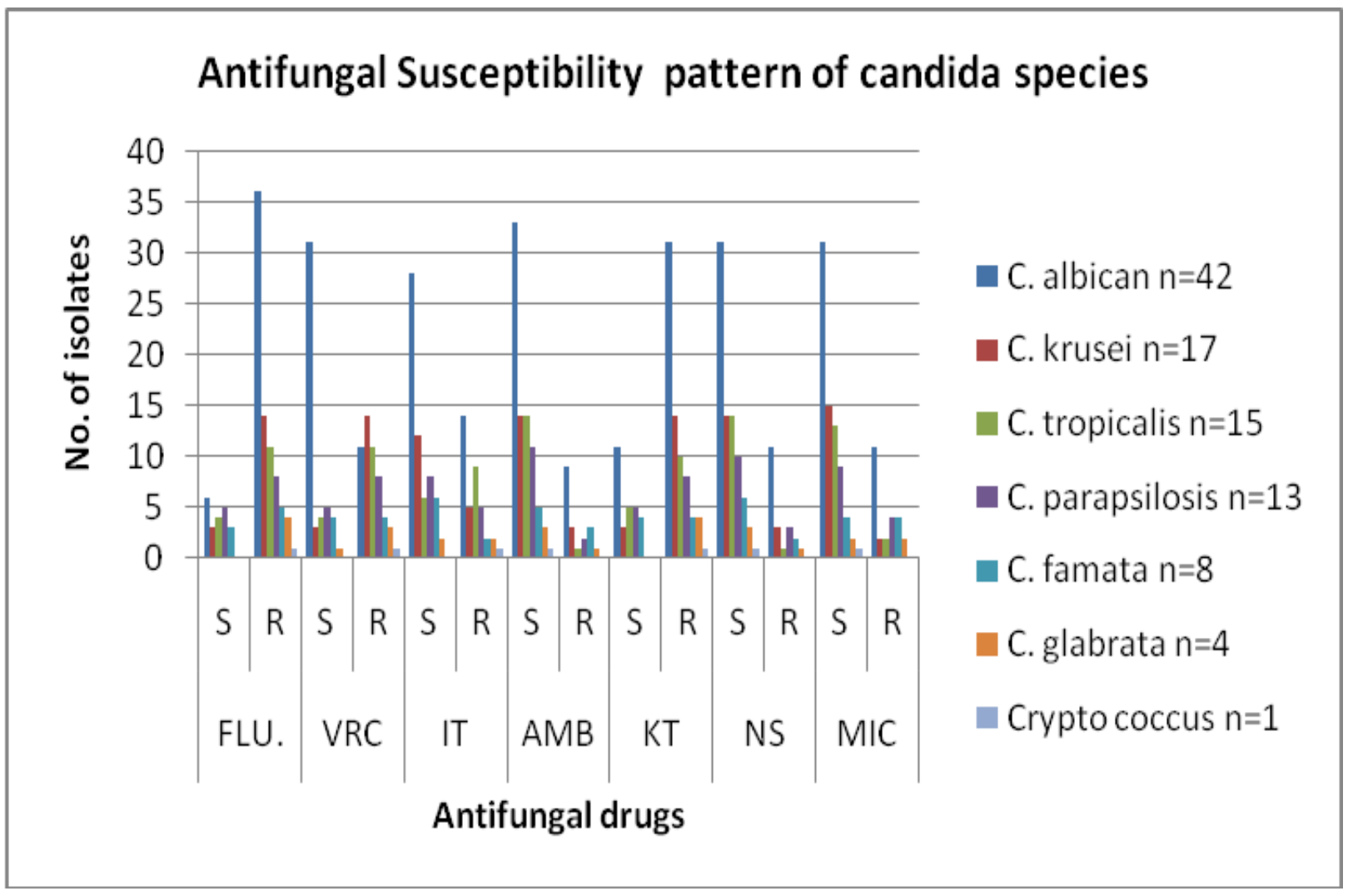


Table.5 Antifungal Susceptibility of Candida species

\begin{tabular}{|c|c|c|c|c|c|c|c|c|c|c|c|c|c|c|}
\hline \multirow[t]{2}{*}{ Species } & \multicolumn{2}{|c|}{ FLU. } & \multicolumn{2}{|c|}{ VRC } & \multicolumn{2}{|c|}{ IT } & \multicolumn{2}{|c|}{ AMB } & \multicolumn{2}{|c|}{ KT } & \multicolumn{2}{|c|}{ NS } & \multicolumn{2}{|c|}{ MIC } \\
\hline & S & $\mathrm{R}$ & $S$ & $\mathrm{R}$ & $S$ & $\mathrm{R}$ & $\mathrm{S}$ & $\mathrm{R}$ & S & $\mathrm{R}$ & S & $\mathrm{R}$ & S & $\mathrm{R}$ \\
\hline C. alb & $\begin{array}{c}6 \\
4.28 \%)\end{array}$ & $\begin{array}{c}36 \\
(85.71 \%)\end{array}$ & $\begin{array}{c}31 \\
(73.80 \%)\end{array}$ & $\begin{array}{c}11 \\
(26.19 \%)\end{array}$ & $\begin{array}{c}28 \\
(66.66 \%)\end{array}$ & $\begin{array}{c}14 \\
(33.33 \%)\end{array}$ & $\begin{array}{c}33 \\
(78.57 \%)\end{array}$ & $\begin{array}{c}9 \\
(21.42 \%)\end{array}$ & $\begin{array}{c}11 \\
(26.19 \%)\end{array}$ & $\begin{array}{c}31 \\
(73.80 \%)\end{array}$ & $\begin{array}{c}31 \\
(73.80 \%)\end{array}$ & $\begin{array}{c}11 \\
(26.19 \%)\end{array}$ & $\begin{array}{c}31 \\
(73.80 \%)\end{array}$ & $\begin{array}{c}11 \\
(26.19 \%)\end{array}$ \\
\hline C. $k r$ & $\begin{array}{c}3 \\
(17.64 \%)\end{array}$ & $\begin{array}{c}14 \\
(82.35 \%)\end{array}$ & $\begin{array}{c}3 \\
(17.64 \%)\end{array}$ & $\begin{array}{c}14 \\
(82.35 \%)\end{array}$ & $\begin{array}{c}12 \\
(70.58 \%)\end{array}$ & $\begin{array}{c}5 \\
(29.41 \%)\end{array}$ & $\begin{array}{c}14 \\
(82.53 \%)\end{array}$ & $\begin{array}{c}3 \\
(16.64 \%)\end{array}$ & $\begin{array}{c}3 \\
(16.64 \%)\end{array}$ & $\begin{array}{c}14 \\
(82.53 \%)\end{array}$ & $\begin{array}{c}14 \\
(82.53 \%)\end{array}$ & $\begin{array}{c}3 \\
(17.64 \%)\end{array}$ & $\begin{array}{c}15 \\
(88.23 \%)\end{array}$ & $\begin{array}{c}2 \\
(11.76 \%)\end{array}$ \\
\hline C. $t$ & $\begin{array}{c}4 \\
(26.66 \%)\end{array}$ & $\begin{array}{c}11 \\
(73.33 \%)\end{array}$ & $\begin{array}{c}4 \\
(26.66 \%)\end{array}$ & $\begin{array}{c}11 \\
(73.33 \%)\end{array}$ & $\begin{array}{c}6 \\
(40 \%)\end{array}$ & $\begin{array}{c}9 \\
(60 \%)\end{array}$ & $\begin{array}{c}14 \\
(93.33 \%)\end{array}$ & $\begin{array}{c}1 \\
(6.66 \%)\end{array}$ & $\begin{array}{c}5 \\
(33.33 \%)\end{array}$ & $\begin{array}{c}10 \\
(66.66 \%)\end{array}$ & $\begin{array}{c}14 \\
(93.33 \%)\end{array}$ & $\begin{array}{c}1 \\
(6.66 \%)\end{array}$ & $\begin{array}{c}13 \\
(86.66 \%)\end{array}$ & $\begin{array}{c}2 \\
(13.33 \%)\end{array}$ \\
\hline$C .1$ & $\begin{array}{c}5 \\
(38.46 \%)\end{array}$ & $\begin{array}{c}8 \\
(61.53 \%)\end{array}$ & $\begin{array}{c}5 \\
(38.46 \%)\end{array}$ & $\begin{array}{c}8 \\
(61.53 \%)\end{array}$ & $\begin{array}{c}8 \\
(61.53 \%)\end{array}$ & $\begin{array}{c}5 \\
(38.46 \%)\end{array}$ & $\begin{array}{c}11 \\
(84.61 \%)\end{array}$ & $\begin{array}{c}2 \\
(15.38 \%)\end{array}$ & $\begin{array}{c}5 \\
(38.46 \%)\end{array}$ & $\begin{array}{c}8 \\
(61.53 \%)\end{array}$ & $\begin{array}{c}10 \\
(76.92 \%)\end{array}$ & $\begin{array}{c}3 \\
(23.075)\end{array}$ & $\begin{array}{c}9 \\
(69.235)\end{array}$ & $\begin{array}{c}4 \\
(30.76 \%)\end{array}$ \\
\hline C. $f$ & $\begin{array}{c}3 \\
(37.5 \%)\end{array}$ & $\begin{array}{c}5 \\
(62.5 \%)\end{array}$ & $\begin{array}{c}4 \\
(50 \%)\end{array}$ & $\begin{array}{c}4 \\
(50 \%)\end{array}$ & $\begin{array}{c}6 \\
(75 \%)\end{array}$ & $\begin{array}{c}2 \\
(25 \%)\end{array}$ & $\begin{array}{c}5 \\
(62.5 \%)\end{array}$ & $\begin{array}{c}3 \\
(37.5 \%)\end{array}$ & $\begin{array}{c}4 \\
(50 \%)\end{array}$ & $\begin{array}{c}4 \\
(50 \%)\end{array}$ & $\begin{array}{c}6 \\
(75 \%)\end{array}$ & $\begin{array}{c}2 \\
(25 \%)\end{array}$ & $\begin{array}{c}4 \\
(50 \%)\end{array}$ & $\begin{array}{c}4 \\
(50 \%)\end{array}$ \\
\hline C. gl & $\begin{array}{c}0 \\
(0 \%)\end{array}$ & $\begin{array}{c}4 \\
(100 \%)\end{array}$ & $\begin{array}{c}1 \\
(25 \%)\end{array}$ & $\begin{array}{c}3 \\
(75 \%)\end{array}$ & $\begin{array}{c}2 \\
(50 \%)\end{array}$ & $\begin{array}{c}2 \\
(50 \%)\end{array}$ & $\begin{array}{c}3 \\
(75 \%)\end{array}$ & $\begin{array}{c}1 \\
(25 \%)\end{array}$ & $\begin{array}{c}0 \\
(0 \%)\end{array}$ & $\begin{array}{c}4 \\
(100 \%)\end{array}$ & $\begin{array}{c}3 \\
(75 \%)\end{array}$ & $\begin{array}{c}1 \\
(25 \%)\end{array}$ & $\begin{array}{c}2 \\
(50 \%)\end{array}$ & $\begin{array}{c}2 \\
(50 \%)\end{array}$ \\
\hline $\begin{array}{l}\text { Cryptoc } \\
\mathrm{n}=1\end{array}$ & $\begin{array}{c}0 \\
(0 \%)\end{array}$ & $\begin{array}{c}1 \\
(100 \%)\end{array}$ & $\begin{array}{c}0 \\
(0 \%)\end{array}$ & $\begin{array}{c}1 \\
(100 \%)\end{array}$ & $\begin{array}{c}0 \\
(0 \%)\end{array}$ & $\begin{array}{c}1 \\
(100 \%)\end{array}$ & $\begin{array}{c}1 \\
(100 \%)\end{array}$ & $\begin{array}{c}0 \\
(0 \%)\end{array}$ & $\begin{array}{c}0 \\
(0 \%)\end{array}$ & $\begin{array}{c}1 \\
(100 \%)\end{array}$ & $\begin{array}{c}1 \\
(100 \%)\end{array}$ & $\begin{array}{c}0 \\
(0 \%)\end{array}$ & $\begin{array}{c}1 \\
(100 \%)\end{array}$ & $\begin{array}{c}0 \\
(0 \%)\end{array}$ \\
\hline Total $n=100$ & $\begin{array}{c}21 \\
(21 \%)\end{array}$ & $\begin{array}{c}79 \\
(79 \%)\end{array}$ & $\begin{array}{c}48 \\
(48 \%)\end{array}$ & $\begin{array}{c}52 \\
(52 \%)\end{array}$ & $\begin{array}{c}62 \\
(62 \%)\end{array}$ & $\begin{array}{c}38 \\
(38 \%)\end{array}$ & $\begin{array}{c}81 \\
(81 \%)\end{array}$ & $\begin{array}{c}19 \\
(19 \%)\end{array}$ & $\begin{array}{c}28 \\
(28 \%)\end{array}$ & $\begin{array}{c}72 \\
(72 \%)\end{array}$ & $\begin{array}{c}79 \\
(79 \%)\end{array}$ & $\begin{array}{c}21 \\
(21 \%)\end{array}$ & $\begin{array}{c}75 \\
(75 \%)\end{array}$ & $\begin{array}{c}25 \\
(25 \%)\end{array}$ \\
\hline $\mathbf{x} 2$ & 0.3 & 613 & 0.0 & 009 & 0.3 & 743 & 0.65 & 927 & 0.45 & 512 & 0.79 & 999 & 0.2 & 963 \\
\hline
\end{tabular}


These isolates were further confirmed by germ test tube, microscopic examination of Corn Meal Agar growth, Sugar Fermentation Test and Sugar Assimilation Test and other biochemical test and confirmed the identification patterns of Candida species (Fig. 1, 2, 3, 4, 5). These indicate that Hichrom agar can be used at field level for rapid presumptive identification. This medium also carries the potential of improving identification of Candida from mixed culture.

To conclude the present study showed that Candida albicans was the most commonly isolated yeast from various clinical specimens and also the increase in the resistance especially to azole is a major concern.

Therefore the species level identification of Candida isolates and its sensitivity profile is must. More importantly this capability will also enable clinician to choose appropriate antifungal agent, this decreasing patient morbidity and mortality.

\section{References}

Abi-said D, Anaissie E, Uzun O, Raad I, Pinzcowski $\mathrm{H}$, Vartivarian $\mathrm{S}$. The epidemiology of hematogenous candidiasis caused by different Candida species. Clin infect Dis 1997; 24:1112-28.

Ajello L and R.J. Hay, 1997. Medical mycology Vol 4 Topley and Wilson, microbiology and infections.9th edition, Arnold London.

Ali Zarei Mahmoudabadi, MajidZarrin, Maryam BeheshtiFard. Antifungal Susceptibility of Candida Species Isolated From Candiduria. Jundishapur J Microbiol. 2013;6(1): 24-28. DOI: 10.5812/jjm.4633.

Anil K Paswan, Dinesh C.Raju, D.K. Singh, Sandeep Khuba, R.K. Dubey.
Isolation and distribution of Candida species among different clinical situations in critically ill patients: prospective study IJBR 3[02] [2012]120- 126.

Anil S and Challacomve SJ. Oral lesions of HIV and AIDS in Asia: An Overview Oral Disease. 1997:3(1):36-40

Enwuru, C.A., A Ogunledun, N Idika, NV Enwuru, F Ogbonna, M Aniedobe, et al., Fluconazole resistant opportunistic oropharyngeal Candida and nonCandida yeast-like isolates from HIV infected patients attending ARV clinics in Lagos, Nigeria. Afr Health Sci 2008; 8(3): 14248.

Koneman E, Allen S, Janda W, Procop G, Schreckenberger P, woods G. Colour Atlas and Textbook of diagnostic Microbiology.

Lattif AA, Banerjee U, Prasad R, Biswas A, Wig N, Sharma N, et al., Susceptibility pattern and molecular type of species specific Candida in oropharyngeal lesions of Indian Human Immunodeficiency Viruspositive patients. J. Clin. Microbiol 2004; 42(3): 1260-62.

Mokaddas EM, Al-Sweih NA, Khan ZU. Species distribution and antifungal susceptibility of Candida bloodstream isolates in Kuwait: a 10-year study Indian J Med Microbiol 2007; 56: 255-59.

Nirmaladevi Somsundaram Isolation and Speciation of Candida from Various Clinical Samples in a Tertiary Care Hospital Volume 7 Number 05 (2018)

Ragini A.K., Sandhya Belawadi, GaytriDevi, Indumal. 2001. Characterisation and antifungal susceptibility testing for Candida in a tertiary care hospital. J. Health Sci. Res., 2(2): 112.

Shanoo Sharma Isolation, Speciation and Characterization of Candida Species in Clinical Isolates Volume 6 Number 
7 (2017) pp. 404-413

Shivaprakasha S, Radhakrishnan K, Karim

PM. Candida spp. other than Candida

albicans: a major cause of fungaemia

in a tertiary care centre. Indian J Med
Microbiol 2007; 25(4): 405-07.

Vijaya D, Harsha T.R, Nagaratnamma, 2011. Candida Speciation Using Chrom Agar, Journal of Clinical and Diagnostic Research. 5(4): 755-757.

\section{How to cite this article:}

Seema Surana and Kusumlata Lodha. 2018. Speciation and Antimycotic Susceptibility Pattern of Candida Species Isolated from Various Clinical Specimen by Using Chromogenic Agar and Conventional Method. Int.J.Curr.Microbiol.App.Sci. 7(12): 716-726.

doi: https://doi.org/10.20546/ijcmas.2018.712.088 